\section{Sterben in der Schweiz und kirchliche Palliative Care}

\section{Pascal Mösli}

\section{Einleitung}

Im November letzten Jahres wurden die Ergebnisse des Nationalen Forschungsprogramms (NFP) 67 zu Sterben und Tod in der Schweiz präsentiert. Die Forschungen zeigen einerseits spannende Einsichten, welche auch von den Kirchen zur Kenntnis genommen werden sollten. Andererseits fällt bei den Forschungsergebnissen auf, dass das kirchliche Engagement für sterbende Menschen und ihre Angehörige nicht vorkommt. Zudem werden die traditionellen, religiösen Ressourcen marginalisiert und sie werden im Gegenüber zu einer sogenannten neuzeitlichen Spiritualität als überholt angesehen.

Der vorliegende Beitrag will beide Aspekte näher beleuchten: Einerseits stellt er einige Herausforderungen für die Kirchen dar, andererseits analysiert er die im NFP 67 ersichtliche kritische Tendenz aktueller Forschung gegenüber Religion und Kirche. Um die beiden Aspekte im kirchlichen Kontext einordnen zu können, beleuchtet er die aktuellen seelsorglich-diakonischen Tätigkeiten und Entwicklungen im kirchlichen Umfeld.

\section{Das nationale Forschungsprogramm zu Sterben und Tod}

Während fünf Jahren ging das NFP 67 Fragen zum Thema Sterben und Tod in der Schweiz nach. In 33 Forschungsprojekten wurden verschiedene Aspekte des Sterbens in der Schweiz untersucht. Ziel des Programms war es, Handlungs- und Orientierungswissen für die letzte Lebensphase zu erarbeiten. Neue Erkenntnisse brachten die Forschungen vor allem hinsichtlich der Versorgungssituation, der Lebensende-
Entscheidungen, der ethischen Grundlagen und rechtlichen Regulierungen sowie der gesellschaftlichen Ideale des guten Sterbens.

Im Porträt des Nationalfondsprojektes lässt sich der Anspruch des Programms ablesen: «Das Nationale Forschungsprogramm 67, das der Bundesrat im Jahr 2010 lanciert hat, soll dazu beitragen, Veränderungen und neu entstehende Bedürfnisse rund um das Sterben besser zu verstehen. Für die Durchführung des Programms hat der Bundesrat 15 Millionen Franken zur Verfügung gestellt. Die Forschungsergebnisse sollen Versorgungslücken und problematische Versorgungssituationen identifizieren und Betroffenen wie auch Behandlungsteams möglichst nützliche Grundlagen für Entscheidungen zur Verfügung stellen. Darüber hinaus sollen Vorschläge für die Anpassung rechtlicher Regelungen entwickelt und ethische Implikationen reflektiert werden. Schliesslich sollen gesellschaftliche Veränderungen in der Wahrnehmung des Lebensendes besser verstanden werden. Bei all dem hat das NFP 67 das Ziel, wissenschaftliche Kompetenzen im Bereich der Lebensende-Forschung in der Schweiz aufzubauen und zu verstärken».1

Dieser Passus zeigt die Bedeutung auf, welcher das NPF 67 im politischen und gesellschaftlichen Kontext hat und macht damit deutlich, dass es auch aus kirchlicher Sicht wichtig ist, die Forschungsergebnisse und deren Interpretationen zur Kenntnis zu nehmen und zu beurteilen.

Wenn man die Forschungsergebnisse studiert, erblickt man eine Fülle von Einsichten, die auch für das kirchliche Handeln am Lebensende interessant und relevant sind. In Kapitel 5 dieses Beitrags sollen Herausforderungen und Perspektiven, die durch die Forschungsergebnisse ersichtlich werden, für kirchliches Handeln am Lebensende entfaltet werden.

1 URL: http://www.nfp67.ch/de/das-nfp, abgerufen am 1. Mai 2018.
Jahrbuch Diakonie Schweiz 2 (2018) - ISSN 2504-3994

Dieser Text ist lizenziert unter einer Creative Commons Namensnennung 4.0 International Lizenz (CC BY 4.0): (https://creativecommons.org/licenses/by/4.0/).
Jahrbuch Diakonie Schweiz 2 (2018) http://dx.doi.org/10.22018/JDS.2018.6 
Wenn man das Forschungsprojekt daraufhin untersucht, was die Kirchen für Menschen am Lebensende bereits tun, findet man wenig. Kein Forschungsprojekt macht das Wirken der Kirchen oder Religionsgemeinschaften direkt zum Thema. Dort, wo spirituelle Aspekte thematisiert werden, geht die Darstellung oft mit einer Distanzierung zu kirchlicher, christlicher bzw. überhaupt religiös institutionalisierter Gemeinschaftlichkeit einher. Der Bericht zum NFP 67 ist von daher auch kritisch daraufhin zu lesen, wie Spiritualität und kirchliches Handeln in der Schweiz wahrgenommen wird. Hierzu versucht Kapitel 4 einige Bemerkungen beizusteuern.

Zuerst soll jedoch im nächsten Kapitel 3 im Sinne einer kurzen Übersicht zur Darstellung kommen, in welchen Feldern kirchliches Engagement für Sterbende und ihre Angehörigen wie auch für die Menschen im Gesundheitswesen heute wirksam ist. Ein Engagement, das, wie bereits erwähnt, in den Forschungsprojekten keinerlei Resonanz hat.

\section{Kirchliches Handeln im Kontext von Palliative Care}

a) Seelsorge im stationären, institutionellen Kontext

In den meisten grösseren und grossen Spitälern der Schweiz sind universitär ausgebildete, kirchliche Seelsorgende verbindlich bei der Betreuung der Patientinnen und Patienten eingebunden. An den meisten Orten wird ökumenisch zusammengearbeitet, die Finanzierung erfolgt durch die Kantonalkirchen, manchmal auch durch die Gesundheitseinrichtungen selbst. Die Seelsorgenden arbeiten eng mit den Betreuungsteams zusammen und sind dabei meist mit einem Schwerpunkt in den Palliativabteilungen tätig. Sie bieten ihre Dienste nicht nur den Betroffenen und den Angehörigen, sondern auch den im Gesundheitswesen tätigen Fachleuten und Teams an.

Die enge interprofessionelle Zusammenarbeit schlägt sich auch in der Fachorganisation palliative.ch nieder, in welcher die Seelsorge, seit zwei Jahren als Fachgruppe organisiert, fachliche Standards im palliativen
Kontext erarbeitet. Zudem wurden in einer Task Force die Standards für Spiritual Care entwickelt, welche für die verschiedenen Berufsgruppen im Bereich der Palliative Care gelten sollen.

Weniger einheitlich ist die Situation in den Institutionen der Langzeitpflege. Während in den grossen Kantonen (wie beispielsweise Bern, Zürich und Waadt) Kennzahlen festgelegt wurden, gemäss denen die seelsorgerlichen Ressourcen in den Institutionen eingerichtet sind oder eingerichtet werden sollten, ist dies in vielen anderen Kantonen nicht oder noch nicht der Fall. Die vom Bundesamt für Gesundheit geforderte PalliativeCare-Spezialisierung der Langzeitinstitutionen hat zur Folge, dass an manchen Orten Türen für die kirchliche Seelsorge aufgehen, um sich im Kontext der spirituellen Dimension von Gesundheit - noch besser und verbindlicher in den Institutionen zu integrieren. Doch noch nicht überall, werden diese Türen auch durschritten, will heissen, nicht überall werden die religiös-spirituelle Dimension sowie die Zusammenarbeit mit der Seelsorge in den palliativen Konzepten der Langzeitinstitutionen verankert.

Für das Selbstverständnis der Seelsorge in Spitälern, Langzeitinstitutionen und Palliativstationen ist zentral, dass sie sich nicht an kirchlicher Mitgliedschaft der Betroffenen orientiert, sondern weltanschaulich offen allen Menschen ihre Dienste anbietet. Sie ist dabei besonders sensibel für die Vulnerabilität der Betroffenen, was sich in der Sterbebegleitung u.a. darin äussert, dass sie Menschen auch dann seelsorglich begleitet, wenn sie sich verbal nicht mehr äussern können oder im Koma liegen.

Die Kirchen stehen in den nächsten Jahren im stationären, institutionellen Kontext vor einigen Herausforderungen:

- An einigen Orten sollte die ökumenische Zusammenarbeit verbindlicher ausgestaltet werden; dass je ein reformierter und ein katholischer Seelsorger dieselbe Abteilung besuchen, wird heute von den Gesundheitsfachleuten kaum mehr verstanden und bindet Ressourcen am falschen Ort; 
- an vielen Orten sind die Ressourcen, besonders in der Langzeitpflege, für die Seelsorge ungenügend und müssten ausgebaut werden; neue Finanzierungsmodelle, bei denen Institutionen seelsorgliche Leistungen mitfinanzieren, müssten entwickelt werden;

- das Profil der Seelsorge ist für viele Fachleute im Gesundheitswesen nicht (mehr) klar; damit fehlt an einigen Orten das Verständnis, um interprofessionell verbindlich zusammenarbeiten zu können;

- im klinischen Kontext wird zunehmend das interprofessionelle Konzept der Spiritual Care wichtig, welches die spirituelle Betreuung als Aufgabe aller Professionen auffasst; das Konzept sollte von der Seelsorge bewusst aufgegriffen werden, um die spirituelle Dimension bei der Betreuung zu stärken und ihre eigene Rolle als Prozessmoderator und Spezialist für diese Dimension einzubringen;

- im klinischen Kontext beschäftigen sich alle Professionen mit der Weiterentwicklung ihrer Disziplinen durch Forschung; die Seelsorge tut dies noch sehr wenig, aus den Felder konkreter seelsorglicher Praxis sollten mehr Impulse für die universitäre Forschung kommen;

- die Zusammenarbeit mit Zuständigen der Kirchgemeinden und Pfarreien muss vielerorts noch verbindlicher gestaltet werden; es braucht eine gemeinsame Vision seelsorglicher Begleitung im stationären und ambulanten Feld und dafür wiederum braucht es Gefässe der Kommunikation und Zusammenarbeit zwischen Seelsorgenden in Institutionen und Gemeinden;

- der Einbezug von Begleitern nichtchristlicher Religionen gerade für sterbende Menschen müsste verbindlicher ausgestaltet werden; christliche Seelsorge hat für die spirituelle Begleitung in öffentlichen Institutionen einen gesellschaftlichen Vertrauensvorschuss und ein Privileg, das sie dazu nutzen sollte, um Zuständi- ge nichtchristlicher Religionsgemeinschaften in verbindlichen und geregelten Formen in der Begleitung einzubinden.

b) Seelsorge, sozialdiakonische und ebrenamtliche Begleitung im ambulanten Kontext bzw. in den Kirchgemeinden und kirchlichen Regionen

Zu einer der zentralen Aufgaben der Pfarrerinnen und Pfarrer gehört die Begleitung kranker und sterbender Menschen und ihrer Angehörigen. Zudem gestalten sie Abdankungsfeiern und begleiten die Angehörigen auch nach dem Todesfall weiter. In der Begleitung Kranker und Sterbender engagieren sich vielerorts aber auch Freiwillige. In einigen Kantonen gibt es regionale Angebote der Begleitung durch Freiwillige (z.B. Aargau, Zürich und Zentralschweiz), zudem existieren auch einige Fortbildungsprogramme für die Freiwilligen bei Palliative Care (Aarau, Appenzell). In der Nachsorge sind neben der individuellen Begleitung Trauernder vielerorts auch Trauergruppen tätig. Auch die kirchliche Sozialarbeit hat es in der Altersarbeit und überhaupt in ihrer Beratungstätigkeit oft mit Menschen zu tun, welche persönlich oder wegen ihrer Angehöriger in eine palliative Situation verwickelt sind.

Dabei ist insbesondere in ländlichen Gebieten die Zusammenarbeit mit anderen Akteuren der Palliative Care wie etwa den Hausärztinnen und Hausärzten und Spitexfachpersonen teilweise eingerichtet. Man kennt sich und zieht sich bei Bedarf bei. Doch diese Zusammenarbeit ist an vielen Orten kaum oder noch zu wenig etabliert. Das hat verschiedene Gründe: Die Spitexorganisationen haben sich vergrössert und sind oft nicht deckungsgleich mit den Organisationseinheiten der Kirchgemeinden; es entstehen Ärztezentren mit regionaler Ausstrahlung; es gibt eine hohe Fluktuation bei den Mitarbeitenden der Gesundheitsorganisationen, welche längerfristige Beziehungen erschweren und bei vielen Gesundheitsfachleuten sind die kirchlichen Angebote und vor allem das kirchliche Profil von heute wenig bekannt. 
Einige Kantonalkirchen haben Fachgruppen, Koordinations- und Projektstellen für Palliative Care eingerichtet. Dabei sind kantonale Konzepte entstanden, welche u.a. zum Ziel haben, die regionale und lokale Vernetzung der Kirchgemeinden mit den Gesundheitsakteuren neu zu initiieren oder zu stärken.

Im ambulanten Feld der Kirchgemeinden und kirchlichen Regionen lassen sich folgende Herausforderungen erkennen:

- Die erwähnte Zusammenarbeit mit den Gesundheitsfachleuten ist elementar. Wenn diese die Angebote der Kirchen und vor allem auch die dahinter stehenden Personen kennen und schätzen, können sie Botschafter für die Kirche sein, indem sie Kranke, Sterbende und ihre Angehörigen auf die kirchlichen Angebote aufmerksam machen. Andererseits sind viele der Gesundheitsfachleute selbst an religiösen und spirituellen Fragen und darum auch am Dialog mit den kirchlichen Mitarbeitern interessiert. Grundlage für diese Zusammenarbeit bildet eine möglichst einfache und verlässliche Kommunikation.

- Bestattungen, eine der traditionellen Aufgabenfelder der Pfarrpersonen, werden vermehrt von anderen Berufsgruppen - wie etwa freiberuflichen Trauerrednern oder Bestattern - durchgeführt. Die Zusammenarbeit mit den Gesundheitsfachleuten führt dazu, dass Pfarrpersonen früh(er) in die Begleitung Sterbender einbezogen werden. So wird auch die Bestattung selbst, als sinnvoller und beziehungsgesättigter Abschluss der Begleitung Aufgabe der Pfarrpersonen bleiben.

- Die interne Zusammenarbeit in den Kirchgemeinden ist wichtig. Wie sind die Aufgaben innerhalb den Angestellten einer Kirchgemeinde aufgeteilt, welche Aufgaben übernehmen die kirchlichen Sozialarbeiter und welche die Pfarrpersonen, wie wird die Kommunikation gestaltet, wer kümmert sich um die Freiwilligen, wie wird die Bereitschaft organisiert?
- Die palliative Versorgung im ambulanten Feld entwickelt sich zurzeit intensiv weiter. Es entstehen mobile palliative Dienste, welche die ambulante Versorgung verbessern. Zudem sollen die Informationen einheitlicher über gemeinsame Behandlungspläne und elektronische Behandlungsdossiers unter den Gesundheitsfachleuten ausgetauscht werden können. Für die Seelsorge ist es wesentlich, sich in diesen neuen Versorgungsstrukturen gut zu integrieren.

- Die «sorgende Gemeinschaft» (Caring Community), d.h. die Entwicklung einer gemeindlichen Sorgekultur etabliert sich als wichtiges Konzept in der palliativen, ambulanten Versorgung. Der Begriff der «sorgenden Gemeinschaft» etabliert sich langsam als politisch aufgegriffener Leitbegriff, für eine neue Weise, «sozial-staatliche Verantwortung und lokales Engagement miteinander zu verbinden» (Thomas Klie). Kirche mit ihren ausdifferenzierten geistlichen, seelsorglichen, diakonischen und kulturellen Aktivitäten hat die Möglichkeit und Chance, in den entstehenden Netzwerken zu kooperieren, neue Partner zu finden und ihre bestehenden Ressourcen auch dort einzubringen, wo sie noch nicht genutzt werden.

- Falls der Ausbau der palliativen Unterstützung zuhause politisch weiterverfolgt bzw. unterstützt wird, wird auch die Wichtigkeit des Engagements von Freiwilligen zunehmen. Sie kümmern sich um Sterbende, aber auch um die oft vernachlässigte Gruppe der sorgenden Angehörigen. Hier spielt die Kirche oft eine wichtige Rolle und kann diese noch weiter ausbauen. Wie Erfahrungen etwa aus dem Kanton Aargau zeigen, hat das Engagement von Freiwilligen auch einen positiven Effekt auf die Einbindung der professionell tätigen Seelsorgenden in der Begleitung.

- Um die kirchgemeindlichen palliativen Sorgeräume verbindlich einrichten zu können, bedarf es einer Sensibilisierung der kirchlichen Mitarbeitenden einerseits und der organisatorischen Verankerung des palliativen Anliegens in den gemeindlichen und re- 
gionalen Leitungsgremien andererseits. Damit kann verhindert werden, dass das palliative Thema in den Gemeinden vom Engagement einzelner Personen abhängig ist.

\section{Kritische Bemerkungen zum NFP 67 aus kirchlicher Sicht}

Es fällt auf, dass im Bericht zu den Forschungsergebnissen das Konzept gemeinschaftlicher Religion durch dasjenige persönlicher Spiritualität ersetzt wird. Es wird vorausgesetzt, dass «neue spirituelle Angebote für Sterbende traditionelle christliche Vorstellungen, die zunehmend an Bedeutung verlieren» ${ }^{2}$ ablösen. Dazu passt, dass die Bedeutung kirchlicher Seelsorge und sozialdiakonischen Handelns, wie sie im Kapitel 3 kurz umrissen wurden, kaum erwähnt wird und es kein Forschungsprojekt gibt, welches kirchliches Handeln zum Thema hat. Obwohl die Bedeutung der ambulanten Versorgung Sterbender und ihrer Angehöriger im Bericht hervorgehoben wird, spielen die mannigfaltigen kirchlichen Angebote im ambulanten, kirchgemeindlichen Kontext keine Rolle. Dabe wird nicht zur Kenntnis genommen, dass sich kirchliche Seelsorge längst als Teil von Spiritual Care versteht und sich seelsorglich-diakonisches Handeln selbstverständlich auf die persönlichen Bedürfnisse der Betroffenen einstellt und ihre Suchbewegungen unterstützt. Die Unterscheidung von Spiritualität, die als die moderne Form der Sinnsuche aufgefasst wird, gegenüber Religion, welche als traditionell und damit überholt qualifiziert wird, hat weitreichende Folgen. In einem Interview mit dem Fachportal diakonie.ch meint etwa der Theologe Prof. Dr. Markus Zimmermann, der die Leitungsgruppe des Nationalforschungsprogramms präsidierte: Es zeigt sich, «dass es nicht um kirchliche Tradition geht in Gesprächen am

2 Schweizerischer Nationalfonds (SNF), Synthesebericht NFP 67 Lebensende, Bern 2017,9.
Lebensende, sondern um das, was geschieht im Sterbenden. Deshalb sollten Seelsorgende nicht gebunden sein an etwas Bestimmtes. In einem Sterbenden tauchen die Bilder auf, die auftauchen wollen. Wir sollten uns alle von fixen Vorstellungen und Bildern lösen, was den Sterbeprozess angeht. Ich finde das sehr schön: Wenn das Sterben konkret wird, gibt es eine grosse Offenheit. ${ }^{3}$ Der Subtext in diesen Sätzen lautet: Das, was beim Sterbenden geschieht, steht im Gegensatz zu kirchlichen Traditionen bzw. die kirchlichen Traditionen haben mit den Erfahrungen der Sterbenden nichts zu tun. Fazit: Man muss sich von ihnen lösen, damit man sich auf die Offenheit des Sterbens einlassen kann. Dahinter steckt ein bestimmtes, tendenziell autoritäres Bild von kirchlicher Tradition. Diese wird vorgestellt wie ein fixes Glaubenssystem, das konkreten, aktuellen Lebenserfahrungen gegenübersteht. Der Mensch muss dann irgendeine Brücke bauen, um sein Leben mit «der» Tradition zu verbinden bzw. er muss seine Erfahrungen irgendwie in die Tradition einordnen oder dieser vielleicht sogar unterordnen. Doch Tradition lässt sich, biblisch begründet, ganz anders verstehen: Als das Aufspüren einer geistlichen, spirituellen Dimension gerade mitten in den aktuellen Lebenserfahrungen; ein Aufspüren, das freilich nicht auf sich selbst angewiesen bleibt, sondern im Dialog steht mit den Erfahrungen vieler Menschen, die vor uns da waren. Auch der Sterbeprozess des Einzelnen ist eingebettet in die unzähligen Geschichten von früheren Sterbeerfahrungen und Deutungen und steht damit in einer Tradition zu deren Erzählungen. Zugleich ist das Sterben des Einzelnen einzigartig und in diesem Sinne ein offener Prozess, der selbst die Tradition aktualisiert und weiterschreibt. Darum ist es weder hilfreich noch sachgemäss, wenn man Religion und Spiritualität auseinanderdividiert - beide gehören zusammen. Während Religion tendenziell die gemeinschaftliche, dialogische

3 URL: https://www.diakonie.ch/wir-sollten-uns-von-fixen-vorstellungen-und-bildern -loesen-was-den-sterbeprozess-angeht/, abgerufen am 1. Mai 2018. 
Dimension fokussiert, hat Spiritualität die persönliche Erfahrung des Einzelnen im Blick. Und wie die Erfahrung und Deutung des Einzelnen ohne all die Erfahrungen und Deutungen anderer Menschen nicht denkbar ist, ist auch Religion ohne die persönliche Erfahrung Einzelnen undenkbar. Es erstaunt darum nicht, dass die religiösen Traditionen (nicht nur das Christentum) im Bericht entweder marginalisiert (wie die christliche Tradition) oder gar nicht erwähnt (andere religiöse Traditionen) werden. Damit bleibt aber eine wesentliche Ressource für viele sterbende Menschen und ihre Angehörigen aussen vor. Auch hier werden die vielen Forschungen nicht zur Kenntnis genommen, welche die Bedeutsamkeit religiöser Ressourcen beim Sterben (aber nicht nur dort!) nachweisen.

Zwei weitere kritische Aspekte seien noch erwähnt:

- Die Selbstbestimmung spielt im Bericht (berechtigterweise) eine grosse Rolle, viele Forschungsprojekte befassen sich mit ihr. Doch der Prozess des Sterbens ist im wesentlichen ein unverfügbares Geschehen, bei welchem neben der Selbstbestimmung noch andere Haltungen und Dimensionen wichtig sind: So beispielsweise das Anerkennen der existentiellen Bedürftigkeit und des Angewiesenseins auf andere, also pathische Qualitäten.

- Spiritualität wird im gegenwärtigen Konzept der Palliative Care (vgl. die Definitionen der $\mathrm{WHO}$ und des BAG) als Dimension von Gesundheit verstanden, welche alle Prozesse (auch die medizinischen, pflegerischen usw.) durchdringt. Im Bericht wird Spiritualität eher als ein besonderes, partielles Angebot der Versorgung aufgefasst. Das ist ein Rückschritt gegenüber dem internationalen Diskurs über Spiritualität, welche diese als integrale Dimension der Versorgung von Menschen ansieht.

\section{Herausforderungen für kirchliches Handeln bei Palliative Care}

Selbstkritisch bemerkt zeigt der Forschungsbericht auf, dass es offensichtlich noch nicht gelungen ist, kirchliche Konzepte und kirchliches Handeln so im professionellen und öffentlichen Diskurs einzubringen, dass sie als Teil einer gemeinsamen Sorge (im Sinne der interprofessionellen Spiritual Care und der Caring Communities) um die spirituellen und existentiellen Bedürfnisse Sterbender und ihrer Angehörigen wahrgenommen werden. Daraus ergeben sich einige Herausforderungen für die Diakonie:

- In der ambulanten Versorgung sollte die Vernetzung zwischen Akteuren der Kirchen und den Gesundheitsfachleuten (insbesondere Spitex und Hausärzte) initiiert oder vertieft werden. Kirchliche Akteure müssen dafür auf die Gesundheitsversorger zugehen, ihr Angebot und ihr Profil erklären und die Zusammenarbeit verbindlich gestalten.

- Die kirchlichen Akteure (Pfarrerinnen und Sozialdiakone) sollten für die Zusammenarbeit im Feld der Palliative Care sensibilisiert und gebildet werden. Profil, Kompetenzen und Angebot der Berufsgruppen müssen geklärt und in einer für andere Gesundheitsprofessionen verständlichen Sprache beschrieben und kommuniziert werden.

- Die Veränderungen der Sterbeprozesse, die durch Forschungen des NFP 67 aufgezeigt werden konnten (Zunahme tiefer Sedierung, Demenz usw.) sollten bei der Weiterentwicklung seelsorglicher Konzepte und Fortbildungen berücksichtigt werden.

- Die Bedeutung der Selbstbestimmung wurde durch verschiedene Forschungsprojekte des NFP 67 für verschiedene Zielgruppen konkretisiert. Die Kirchen sollten sich in den gesellschaftlichen Diskurs mit ihren religiösen Anliegen und Perspektiven aktiver einbringen und sich zugleich als Partner in der Unterstüt- 
zung der Selbstbestimmung der Bürger anbieten (z.B. im Bereich des Advance Care Planning).

- Die Unterstützung von sterbenden Menschen und insbesondere auch der pflegenden Angehörigen wird künftig ohne freiwillig engagierte Menschen nicht zu bewerkstelligen sein. Die Kirchen sollten sich mit ihrer grossen Erfahrung in der Freiwilligenarbeit aktiv einbringen, die Vernetzung fördern und eigene Freiwilligenprojekte ausbauen.

- Der Bericht endet mit dem Desiderat, die Sterbeforschung zu anerkennen und zu intensivieren. Diese trage dazu bei, «Veränderungen besser zu verstehen, Konsequenzen für die medizinische, politische, wirtschaftliche und rechtliche Praxis zu reflektieren». ${ }^{4}$ Damit auch die religiösen und spirituellen Anliegen im Fokus sind, müssen sich kirchliche Akteure selbst mit Forschungsprojekten in den Diskurs einbringen. Dies geschieht bis heute leider noch viel zu wenig.

Autor:

Pascal Mösli,

Theologe und Supervisor MAS, Beauftragter Spezialseelsorge und Palliative Care der Reformierten Kirchen Bern-Jura-Solotburn und Dozent für Ethik und Spiritual Care im Gesundheitswesen, Mitglied des Forschungsteams Spiritual Care in Zürich

4 Schweizerischer Nationalfonds (SNF), Synthesebericht (Anm. 2), 47.

Jahrbuch Diakonie Schweiz 2 (2018) - ISSN 2504-3994 